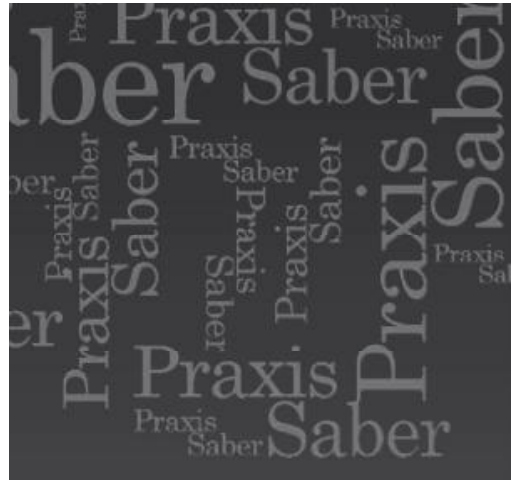

Julio Fernando Acosta Muñoz

Docente Escuela de Psicología

Psicólogo, Magíster en Educación

Universidad Pedagógica y

Tecnológica de Colombia

jufeacmu@hotmail.com

\title{
LAS ACTITUDES INVESTIGATIVAS EN LA FORMACIÓN ESCOLAR
}

\section{Resumen}

Este trabajo es un artículo de reflexión, producto de la investigación denominada 'Actitudes investigativas de los jóvenes dentro de la formación en investigación en la escuela'. El campo de interés está centrado en elaborar el contraste de tipo teórico y crítico frente a la formación investigativa, desde la propuesta de diferentes actitudes investigativas en los procesos formativos de la escuela. Metodológicamente se construye desde la revisión teórica de autores, haciendo a su vez una exploración de la problemática. Primeramente se narran las dificultades manifestadas en torno a la formación investigativa y la relación de este tipo de educación con la visión positivista tradicional. Dentro del texto, se hace la propuesta de visibilizar distintas actitudes en la formación investigativa escolar (experiencia de la infancia, conocimiento de sí mismo y condición críticoreflexiva), basadas en la subjetividad del aula, que colocan como objeto de reflexión y acción al educando en su proceso de investigador.

Palabras clave: actitud, investigación, formación, escuela. 


\section{Researching Attitudes In School Training}

\section{Abstract}

This work is a reflection article, product of the research referred to 'Researching Attitudes of Young People in Research Training at the School'. The field of interest is focused on developing the contrast, of theoretical and critical type, facing the research training from the proposal of different research attitudes in the training processes of the school. Methodologically, it is constructed from the theoretical review of authors, exploring the problem at the same time. First the difficulties, expressed about the research training and the relationship of this type of education with traditional positivist view, are described. Within the text, it is proposed to visualize different attitudes in the scholar research training (childhood experience, self-knowledge, and the reflective and critical condition), based on the subjectivity of the classroom, placing the trainee as an object of reflection and action in his/her researcher process.

Key words: Attitude, research, training, school.

\section{Les AtTtTTUdes InVESTIGATRICES DANS LA FORMATION SCOLAIRE}

\section{Résumé}

Ce travail est un article de réflexion, produit de la recherche dénommée «Attitudes investigatrices des jeunes dans la formation en recherche à l'école ». Le champ d'intérêt est concentré sur l'élaboration du contraste de type théorique et critique face à la formation investigatrice, à partir de la proposition de différentes attitudes de recherche dans les processus formatifs scolaires. On construit, d'une manière méthodologique, à partir de la révision théorique d'auteurs, faisant ensuite une exploration de la problématique. Premièrement on narre les difficultés rencontrées 
en ce qui concerne la formation investigatrice et la relation de ce genre d'éducation avec la vision positiviste traditionnelle. Dans ce texte on présente la proposition de rendre visible différentes attitudes, dans la formation investigatrice scolaire (expérience de l'enfance, connaissance de soi-même et condition critique-réflexive), basées sur la subjectivité de la classe, qui présentent l'élève comme objet de réflexion et d'action dans son processus de chercheur.

Mots clés: altitude, investigation, formation, école.

\section{As Atitudes Investigativas na Formação ESCOLAR}

\section{Resumo}

Este é um artigo de reflexão, resultado da pesquisa sob o nome "Atitudes investigativas de jovens num processo de formação em investigação na escola". O campo de interes está centrado em fazer uma comparação teórica crítica na frente da formação investigativa, desde a proposta de diferentes atitudes investigativas nos processos formativos da escola. Quanto à metodologia se constrói desde a revisão teórica de autores, fazendo à sua vez uma exploração da problemática. Narram-se as dificuldades manifestadas em torno à formação investigativa e a relação desse tipo de educação na visão positivista tradicional. Faz-se a proposta de visualizar distintas atitudes na formação investigativa escolar (experiência da infância, conhecimento de sem mesmo e condição crítico-reflexiva), baseada na subjetividade da sala de aula, que colocam como objeto de reflexão e ação ao educando em um processo de pesquisador.

Palavras chave: atitude, investigação, formação, escola. 


\section{Introducción}

Las actitudes investigativas son un fenómeno novedoso poco analizado en el contexto educativo, y más aún en el contexto de la formación en investigación. Este documento está escrito metodológicamente desde el rastreo conceptual frente a los temas relacionados con la formación investigativa, llevando al lector al encuentro de una experiencia educativa donde se reivindica el sitio del estudiante en su proceso de investigador. En este artículo reflexivo, se narran primeramente las dificultades en la formación escolar en investigación, donde prevalecen una serie de sujeciones asociadas a la forma como se "enseña" a investigar, donde se calcan las posturas homogeneizadoras de los paradigmas de la ciencia, colocando estilos investigativos de otros sobre los jóvenes.

A través de la revisión teórica, se construye el significado de la formación en investigación. Este tipo de educación difiere de cualquier otro tipo de educación partiendo del tipo de intensión que dirige este tipo de enseñanza; el propósito de este tipo de formación está en que el alumno introyecte todos los factores, técnicas y procedimientos que involucra la investigación.

Sin embargo este tipo de enseñanza se ve afectada cuando en sus procesos se vincula con la formación positivista tradicional. La formación investigativa positivista tradicional se caracteriza por replicar los modelos y las prácticas dogmáticas de la ciencia sobre los estudiantes, como forma de mantener el control y el conocimiento que se supone como verdad. Este tipo de formación se caracteriza por llevar directamente el conocimiento a los educandos, y por el valor pasivo que terminan asumiendo los escolares frente a su propia formación investigativa.

La propuesta está, entonces, en cómo desde los dispositivos pedagógicos, los estudiantes podrán reconocer en su formación investigativa algo que se va a denominar 'actitudes investigativas', como un ejercicio reflexivo, crítico e innovador permanente sobre sus propias prácticas. Cabe resaltar que este tipo de actitudes difieren al tipo de actitudes que plantean las ciencias "psy", debido a que estas últimas se quedan encasilladas en un concepto "embalsamado" de predisposición.

Las actitudes investigativas que se deben reivindicar en la denominada formación investigativa están basadas en la subjetividad del aula y de 
los educandos. La actitud investigativa como experiencia de la infancia es el modo como representan los educandos su capacidad de asombro y cuestionamiento de todo y por todo, es el espacio y el lugar donde pueden fecundar las más valiosas experiencias educativas como un tipo de experiencia de infancia sin medida, sin proporción, como una infancia escolar y política asociada al aspecto investigativo.

La actitud investigativa como condición crítico-reflexiva está representada en los estudiantes como una mirada constante sobre los actos, los pensamientos, las formas de estar en y con el mundo, en la atención a los fenómenos circundantes en el proceso investigativo, en una relación indisoluble entre el razonamiento y la construcción de juicios, orientada por la libertad de pensar que lleva al desarrollo del ingenio.

La actitud investigativa como conocimiento de sí mismo es la forma como los educandos pueden verse a sí mismos, como una de las formas de comprensión y autoconocimiento que se ha construido por mediación de la reflexión y los dispositivos pedagógicos que atraviesan a todos los escolares. Es así que la actitud investigativa en relación con el conocimiento de sí mismo, se constituye en distintas formas en la actitud general, como una relación con uno mismo, con los otros, con el mundo, y con el inventarse a sí mismo.

\section{Las dificultades en la formación escolar investigativa}

La investigación en el mundo actual es una experiencia social que define el objeto de que se ocupa la investigación, las técnicas, los lineamientos y lógicas donde se desenvuelve la pesquisa. Hablar de investigación implica reflexionar y analizar los cambios y situaciones que ha vivido el pensamiento humano para formar el quehacer de la investigación e investigar, sumado a la permanente reconfiguración de los métodos y las técnicas en investigación, con el surgimiento de nuevos modelos y paradigmas internos que configuran el sentido de investigar y ser investigador.

La sociedad de la información hoy en día está asociada con la experiencia de la investigación, práctica que se manifiesta en las instituciones educativas como un lugar donde los educandos se familiarizan con la lógica de la indagación y su habilidad para investigar, generando de forma sistemática una serie de conocimientos para resolver problemas del contexto. Esta 
actividad ya no es una actividad reservada a la academia o a la universidad: ha entrado en la vida de las escuelas, bajo la premisa de hacer posible que se puedan formar desde la escuela profesionales excelentes, con la iniciación de la investigación en el aula de un colegio.

Es por esto, como parte de la demanda dentro de la globalización pedagógica, que en los últimos años se han asumido una serie de esfuerzos para introducir en las instituciones educativas las condiciones de formación investigativa, constituyendo espacios específicos para la naturaleza y "formación de sujetos investigadores". Lo cierto es que el tema de la denominada formación investigativa se ha vuelto un temaproblema pedagógico, por la relación del rol que puede desarrollar la investigación en el aprendizaje desde la misma indagación y la construcción del nuevo conocimiento. Es así que la escuela asume el anónimo espacio donde se debe aprender a ser investigador, donde el tipo de formación investigativa que adopten los educandos, posee directa relación con el ideal de educación con el que se enseña.

En suma, se puede decir que la educación en Colombia se ha caracterizado por ser predominantemente conservadora, memorista, pasiva e informativa, muy poco centrada en el enseñar a pensar y a resolver problemas de manera creativa. La formación en investigación de los educandos en muchos casos, se ha enfocado a la captación y recolección de información de conocimientos brindados por las "materias duras", haciendo de la enseñanza en investigación un espacio específico para la transmisión de los conocimientos que son "verdaderamente" importantes para la vida laboral del sujeto, encaminados a formar al joven en pro de la sociedad productiva.

Sin embargo, hacia la década de los ochenta en el país, por medio de Colciencias y el Ministerio de Educación, se inició la carrera por relacionar y dar cobertura a la investigación en la escuela. Proyectos como el programa Cucli-Cucli, el proyecto Atlántida, Nautylus, Pléyade, y en la última década el proyecto ONDAS, han buscado y realizado esfuerzos educativos por favorecer la formación del pensamiento científico en los jóvenes en edad escolar.

A pesar de esta voluntad política, gubernamental y pedagógica por inducir la investigación en los escolares, la realidad educativa frente a la formación investigativa es otra: Los docentes en su enseñanza sobre investigación, 
repiten con sus estudiantes las formas como ellos fueron educados en la indagación; asumen las posturas replicadoras y homogeneizadoras de los paradigmas de la ciencia, y los educandos niños, niñas y jóvenes son afectados en la forma como se sobreponen estilos investigativos de otros, es decir, los jóvenes tienen la representación de los sistemas investigativos, que como discurso pedagógico en la formación investigativa, no aparece como el puente entre el saber y el sentido común, sino como el punto de encuentro de ciertos intereses sociales que se materializan en el maestro, las clases y la escuela.

La investigación es una tendencia social hegemónica donde prevalece la relación entre el saber asignado socialmente y la aceptación de esos roles en el juego del poder-saber; relación que se perpetúa aun cuando se cambien las dinámicas pedagógicas-investigativas, lo que hace que se vea muy difícil transformar algo en la escuela, y mas aún en la formación investigativa (Bustamante, 1998).

La formación investigativa en los jóvenes escolares, queda reducida a una situación en la que la vida y la ciencia son separadas en materias o áreas del conocimiento, asumiendo doctrinas como reservas sagradas de principios incuestionables. Es por esto que la escuela se convierte no en el dispositivo "educador", sino inhibidor de las condiciones innatas de investigación. El horizonte del discurso en la investigación y la formación investigativa escolar está en replicar políticas, teorías que obedecen a un sistema productivo, que en los currículos adaptados al nuevo orden social, en las enseñanzas del maestro a sus estudiantes, es donde encuentran su lugar, su sitio.

En algún punto se ha extraviado el horizonte en la educación de la formación investigativa en los escolares: los espacios para el debate, la discusión sobre investigación y formación de investigadores, se han convertido y limitado en lugares para la asesoría de los proyectos de investigación, lugares centrados en qué decir y cómo hacer investigación. El problema de investigar y ser investigador que se vive en la escuela, queda en los extremos de la construcción de conocimientos específicos, como los aprendizajes enfatizados en la metodología o en epistemología de la investigación, ambos paralelos tan reduccionistas: uno por su nivel instrumental y el otro por los posicionamientos tan abstractos, transformando un falso sentimiento de educar el espíritu, la actitud y la actividad investigativa. La relación entre el maestro, el estudiante y 
el saber nuevo, quedan convertidos en recetarios donde se dan simples instrucciones, en el cual el docente le dice al estudiante qué hacer, pero poco se hace por dejar pensar.

Hoy en día investigar es una actividad que ha entrado en la vida de distintas instituciones (organizaciones empresariales, ONGS, entre otras) y de la escuelas, de tal suerte que se afirma que no parece posible que se puedan formar profesionales excelentes sin investigación en el aula de un colegio.

\section{La formación investigativa}

El problema del significado de formar y de la formación dentro del entorno pedagógico, ha constituido distintos horizontes conceptuales sobre estos términos. La educación y los procesos de formación han estado ligados al proceso cultural, como la forma de adquirir o mantener un conocimiento entre el tiempo y las sociedades.

Desde Comenius hasta Kant, pasando por Rousseau y la concepción histórica de Hegel, se plantea la formación como un proceso de humanización de los jóvenes hasta propiciarles la "mayoría de edad", y con ella niveles superiores de autonomía y racionalidad (Flores, 1998). Es así que cuando se habla de formación, es irremediable pensar que el sujeto educado es "hecho por otros", una o más personas se encargan de un modo u otro de su formación. La formación del otro pretende controlar en lo mejor posible, los procesos educativos y actuar sobre el sujeto a educar de modo coherente, concertado y sistemático para su bien máximo (Meirieu, 2003). El hombre es el único ser susceptible de educación [...] el hombre no puede hacerse hombre más que por la educación. No es más que lo que ella hace de él. Y observemos que no puede recibir esa educación más que de otros hombres que a su vez la hayan recibido (Kant, 1980).

Díaz Barriga y Rigo plantean que "la formación se relaciona o se designa de manera fundamental al modo específicamente humano de dar forma a las disposiciones y capacidades del hombre [...], el proceso de formación de una persona lleva implícito el desarrollo de sus potencialidades, las que se pondrán a disposición de otras personas en la relación social cotidiana y en los distintos ámbitos en que convive o se desempeña" (2000: 87).

Sin embargo, cuando se habla de formación investigativa se hace mención y relación a muchos de los aspectos anteriormente citados, pero se 
diferencia notablemente de cualquier otro tipo de educación cuando se habla de los procesos y propósitos que dirigen este tipo de enseñanza, donde el objetivo está en que el educando ensimisme todos los factores que involucran la investigación hacia la formación para la vida.

Moreno (2005) afirma que la formación para la investigación es un proceso que implica diversas prácticas y actores, en el que la intervención de los formadores se concreta en un quehacer académico dirigido a promover y facilitar de manera sistemática los conocimientos, las habilidades, los hábitos, las actitudes y los valores que demanda la realización de la práctica investigativa. Se supone que este proceso posee una intencionalidad, pero no un período temporal definido, pues no se trata de una formación que hay que aprobar antes de hacer investigación. También se accede a dicha formación durante la realización de la investigación y, desde luego, en forma continua a lo largo de toda la trayectoria del sujeto como aprendiz dentro y fuera del sistema escolar.

Esta formación va teniendo distintos matices y se realiza con el apoyo en diversos procedimientos, los cuales tiene que ver con las necesidades y expectativas de los sujetos involucrados en dicha formación. El problema real de la formación en y para la investigación está cuando se determina, se encauza y da "forma" a los escolares con la investigación, desde distintos procesos y actividades que no hacen parte necesariamente de un proyecto concreto de investigación, y se alejan de la naturaleza investigativa, de la búsqueda insaciable del nuevo saber y conocimiento.

Pineda (2008) plantea que la formación en investigación es un lugar nuevo donde el maestro no enseña, no explica nada en forma directa a su educando, sino que es alguien que está atento a la práctica investigativa y ayuda a corregirla, entonces, cuando se trata de aprender, no es él quien dice qué se debe hacer, sino el que enseña a corregir el propio aprendizaje, por tanto, la explicación no es necesaria para remediar una incapacidad de comprensión dentro del proceso de formación investigativa, es todo lo contrario: esta incapacidad es la ficción que estructura la concepción explicadora del mundo; explicar alguna cosa a alguien, es primero demostrarle que no puede comprenderla por sí mismo (Rancière, 2007).

La formación investigativa no es un lugar de entrenamiento donde se aplican unos procedimientos basados en el método científico. La formación de investigadores es la condición donde el estudiante investigador se ve 
como un bricoleur ${ }^{1}$ que selecciona materiales, crea su estudio y, de hecho, se convierte en instrumento de la investigación, desde su propia actitud; es un instrumento de reflexión y no de precisión.

\section{La formación positivista-tradicional y la actitud investigativa}

Uno de los principales factores que ha venido afectando la formación investigativa y la apropiación de una actitud auténtica frente a la investigación en los educandos, es la amalgama que ha tenido este tipo de educación con el positivismo tradicional pedagógico, alejando y abriendo brechas entre la construcción de un conocimiento y la conciencia de una actitud investigativa en los escolares.

La formación positivista tradicional de la investigación se caracteriza por el tipo de relación que se agencia entre sus actores: el aprendiz es visto como algo que hay que desarrollar, enderezar, corregir, adiestrar y disciplinar, es encauzar al estudiante por preceptos, reglamentos y los enunciados de las ciencias donde el maestro es entendido como un

1 El término bricoleur es un término acuñado del término francés bricolage. Desde un nuevo terreno conceptual, según Kincheloe (2005) el bricoleur es un proceso ecléctico que plantea numerosas cuestiones, donde los investigadores deben tratar de mantener la coherencia teórica y la innovación epistemológica en los procesos investigativos. Este carácter multidisciplinario exige un nuevo nivel de investigación, basado en la autoconciencia y el conocimiento de los numerosos contextos en los que cualquier investigador está en funcionamiento. El investigador como bricoleur, destaca la relación entre las formas de ver de un investigador y su historia personal. Apreciando la investigación como un acto de movimiento constante frente a las condiciones del entorno y sí mismo, el investigador como bricoleur abandona la búsqueda de un concepto ingenuo del realismo, centra su lugar en la aclaración de su posición en la red de la realidad y las posiciones sociales de otros investigadores y las formas en que dan forma a la producción y la interpretación de los nuevos conocimientos. En este contexto, la condición del bricoleur es moverse y atacar el dominio de la complejidad, además del descubrimiento de los artefactos invisibles del poder y la cultura, que documentan la naturaleza de su influencia sobre el entorno científico y social. En este proceso, el bricoleur percibe las teorías no como una explicación de la naturaleza, sino como la relación de los sujetos con la naturaleza, para poder construir visiones activas de la metodología de investigación. Por medio del bricoleur, se construyen activamente métodos propios de investigación desde las herramientas que pueden estar a la mano del investigador, en lugar de recibir pasivamente las "correctas", metodologías universalmente aplicables. Es así que el bricolage construye un papel mucho más activo para los seres humanos, tanto en la conformación de la realidad y en la creación de los procesos de investigación y narrativas que lo representan. Tal agencia activa, rechaza las opiniones deterministas de la realidad social que asumen los efectos de determinados procesos sociales, políticos, económicos, y educativos. 
transmisor, el alumno un receptor y la escuela una reglamentación que hay que seguir (Flórez, 1998; Quiceno, 1988). La formación investigativa tradicional se concreta en una función jerarquizante y legitimadora, que lleva a diferenciar a los distintos partícipes que están involucrados en el supuesto proceso de formación de investigadores, maestros, docentes investigadores, semilleros de investigación y tutores, en las relaciones jerárquicas donde el tutor o maestro investigador, representado como un adulto experto, sostiene el crecimiento e incubamiento del saber en sus "semilleros" (Messina, 2011).

Este tipo de enseñanza predispone al estudiante a sujetarse a la comprobación de hechos concretos, demostraciones causales, verificables y racionales del terreno experimental dentro del monismo metodológico, es decir que sólo se puede entender aquello que se considere como una auténtica explicación científica. El objetivo de esta aparente formación investigativa queda relegada a la búsqueda de leyes generales hipotéticas de la naturaleza que subsuman los hechos individuales; el interés dominador del conocimiento y la actitud investigativa tradicional y positivista, está en el énfasis de la predicción de los fenómenos (Giroux, 2004).

Este tipo de formación en investigación, busca homogeneizar y controlar al sujeto de la experiencia, simplificando, cuantificando y reduciendo la realidad a expresiones genéricas y descontextualizadas. Estas metolodogías han permeado desde el análisis de variables e indicadores las ciencias sociales, incluso afectando las formas más alternas de investigación, en el marco de una aparato cultural que ha autodenominado estos modos de ver la realidad como ciencia. Es así que la realidad, la vida social se deshistoriza, quedando reducida a la exposición de los síntomas y efectos de las vidas de los sujetos (Messina, 2011). Paso a paso se va haciendo un trabajo sobre la formación investigativa de los estudiantes, hasta la absorción de los jóvenes a este modelo, llegando al punto en que los alumnos replican "un método científico" como única verdad.

La actitud investigativa dentro de la pedagogía positivista tradicional queda en la tarea indeclinable de llenar a los educandos con los contenidos y retazos de la realidad de los docentes, es una donación de aquellos que se juzgan como sabios a los que califican de ignorantes. Esta educación sublima a los aprendientes a ser siempre subordinados de un poder superior; es por esto que siempre son conducidos a la memorización mecánica del contenido narrado sin entender realmente lo que es, es 
una desvinculación del sujeto con su formación, con su propio ser, con su propia actitud e indagación y cuestionamiento frente al mundo y la realidad (Freire, 1992). Esta formación investigativa queda como una práctica limitante, una teoría educativa urdida para sacrificar los espacios de libertad y autodeterminación del sujeto mediante praxis de adiestramiento y reeducación (Larrosa, 1995), donde la relación maestroalumno, está mediada en el poder que transita a través de la transmisiónconstrucción del saber, donde la pedagogía y la educación son concebidas como dispositivos de poder que garantizan la eficacia en la transmisión del conocimiento (Besley, 2005; Foucault, 2005) .

Esta concepción tradicional mantiene en la práctica una división de los saberes de forma tal que ha llegado a naturalizarse este método hasta llegar a subsistir y perpetuarse. $Y$ es que la escuela tradicional se apoya en ciertas evidencias de sentido común, como el hecho de que la humanidad ha ido produciendo conocimiento oportuno, que se debe conservar y acumular trasmitiéndolo a las nuevas generaciones. Desde esa óptica la función básica de la escuela sería transmitir a esas generaciones los cuerpos de conocimiento disciplinar que constituyen nuestra cultura (Foucault, 2005; Gimeno \& Pérez, 1992).

Las deficiencias sentidas por parte de este modelo en la formación investigativa, han provocado el surgimiento y desarrollo de modelos alternativos, que se preocupan fundamentalmente por indagar el significado de los fenómenos educativos en la complejidad de la realidad natural donde se producen. Sin embargo, según Guba y Lincoln (2000), para la formación bajo otros paradigmas distintos al positivista tradicional, los aprendices de investigador deben ser primero resocializados de "su temprana y generalmente intensa exposición a la perspectiva heredada de la ciencia".

\section{¿Qué son entonces las Actitudes Investigativas?}

El estudio de las actitudes ha sido un elemento que con mucha dedicación ha abordado la Psicología. Rodríguez (1976) afirma que una actitud es una organización duradera de creencias y cogniciones en general, dotadas de una carga afectiva en favor o en contra de un objeto social definido, que predispone a una acción coherente con las cogniciones y afectos relativos a dicho objeto; Secord y Backman (1964) afirman que las actitudes son ciertas regularidades en los sentimientos, pensamientos y predisposiciones a actuar respecto a algún aspecto del entorno. 
Hay puntos en los que es muy difícil poner el límite entre lo conceptual y lo actitudinal, la transmisión de un contenido conceptual va siempre acompañada de un contenido procedimental y un contenido actitudinal. Estas posturas sobre el formar en la investigación a los alumnos, lleva a hacer una reflexión más profunda sobre el tema: la actitud investigativa, no puede quedarse situada sobre los actos predispuestos, sistemáticosformativos, dirigidos y estructurados por sistemas educativos que se alejan cada vez más del verdadero sentido investigativo que todos los sujetos poseen.

Las actitudes investigativas dentro de la formación en investigación, son un aspecto más profundo, que abarca más que un sistema complejo psicológico de conductas dirigidas a la indagación; es una condición que a los ojos de esta reflexión y propuesta teórica y pedagógica, emerge y recoge la experiencia, la infancia, la propia historia, la emotividad, la percepción de cada sujeto, en relación a las condiciones presentes en el entorno, como elementos que se van forjando y consolidando en la escuela: las actitudes investigativas en los jóvenes es algo que se inventa y se reinventa continuamente por mediación de todos los conocimientos, forjando a su vez propias reglas de indagación, rompiendo constantemente con los paradigmas.

Para empezar hay que afirmar que las actitudes de un estudiante frente a la investigación son totalmente distintas a las actitudes investigativas que intentan imponer los adultos en la formación en investigación. Lo que sí hay que analizar es cómo la escuela puede entrever las particulares actitudes de los escolares para su visión como futuros investigadores. Un dispositivo pedagógico será, entonces, cualquier lugar en el que se constituye o se transforma la experiencia de sí. Es cualquier lugar en el que se aprenden o se modifican las relaciones que el sujeto establece consigo mismo (Larrosa, 1995).

La escuela en términos de la construcción diferenciada de las actitudes investigativas, no solo debe mediar y construir una experiencia del mundo exterior "objetiva", debe también hacer que los sujetos escolares encuentren la propia experiencia que tienen los educandos sobre sí mismos y de los otros como sujetos. Aprender a ser investigador y lo que es ser investigador significa desde el dispositivo pedagógico, una constante interrogación consigo mismo, acerca de quiénes son para sí mismos y quién es cada uno de ellos para los demás (Valera, 2001). 
Estas unidades pedagógicas como constitutivos de la subjetividad, adoptan un punto de vista pragmático sobre la experiencia de sí. La pedagogía dentro de las actitudes investigativas no puede ser vista ya como un espacio neutro o problemático de desarrollo o de mediación, como un mero espacio de posibilidades para el desarrollo (Foucault, 1981) o la mejora del autoconocimiento, la autoestima, la autonomía, la autoconfianza, el autocontrol, la autorregulación, etc., sino que produce formas de experiencia de sí en las que los individuos pueden devenir sujetos de un modo particular (Larrosa, 1995).

Disolver lo que aparece tradicionalmente como formación investigativa desde las actitudes investigativas, implica la construcción de un espacio político, pedagógico y educativo diferenciado, donde se visibilicen las nuevas conexiones y diferenciaciones de comportamientos, de movimientos y trayectos, como modos de vínculos que no estén dados a priori. La propuesta de las actitudes investigativas que deben mediar en el dispositivo pedagógico en procura de erosionar las perspectivas dogmáticas y sistemáticas frente a esta formación en la investigación son:

La actitud investigativa como experiencia de la infancia. La experiencia de infancia que se relaciona con la actitud investigativa, se sitúa desde otro orden al instaurado por las ciencias "psy". La actitud de investigar desde la postura de la experiencia de la infancia, es la primera tarea para desnaturalizar lo que se ha denominado como formación investigativa e investigación. Esta actitud se caracteriza por situar el aspecto de la infancia como una parte, un lugar para la construcción del conocimiento donde el sujeto individual o colectivo se vuelve el centro de esta operación (Messina, 2011). La experiencia de la infancia sería el punto donde se quiebra la vida natural, la rutina, la tradición (Larrosa, 2003); la experiencia es un padecer, es un salir de viaje, es salirse de uno mismo e ir hacia lo otro, hacia el otro (Melich, 2002). El camino de la experiencia no implica negar el horizonte libertario de la educación, ni la búsqueda de algo nuevo y más justo, es acercarse a los otros y a sí mismo desde otro lugar, que no es el sitio de la norma, ni la teoría, ni el deber ser (Messina, 2011).

La actitud investigativa como experiencia de la infancia agencia a los jóvenes a que sean potencialidad de cambio, desde su libertad y autonomía del pensamiento. La condición de infancia, con su radical apertura al mundo exterior e interior y con su capacidad de asombrarse y preguntar 
ante todo y por todo, es el espacio y el lugar donde pueden fecundar las más valiosas experiencias educativas e investigativas en los jóvenes. La actitud investigativa desde la perspectiva de la infancia, va más allá del horizonte de la investigación de la niñez. Pues, si bien los actores principales del proceso investigativo son ellos mismos, en realidad, la formación investigativa dirigida por los jóvenes puede referirse a todo lo que ellos consideren relevante, lo que les interese o cualquier cosa que deseen entender mejor (Liebel, 2007).

La experiencia de infancia en la actitud investigativa es aquel estado que en algún momento se ha sido, es una infancia sin medida, sin proporción, una infancia basada en el principio aiónico ${ }^{2}$, el no tiempo. Es una condición investigativa sin límites, donde los jóvenes explotan la espontaneidad, la ingenuidad (Kohan, 2009). Este estado se caracteriza por los actos y espacios creativos, donde los jóvenes mediante la investigación viven lo discontinuo y la experiencia como un acontecimiento que interrumpe, revoluciona, y crean una nueva historia, un nuevo inicio, una actitud investigativa de devenires infantiles.

La actitud investigativa como experiencia de la infancia, es un devenir-niño. Este devenir de por sí juega con otra temporalidad, revoca cualquier calco, cualquier posibilidad de imitación de otras actitudes investigativas, es una condición de cualquier adulto, un niño o ser humano, que se encuentra con aquello que, en principio, "no debería" encontrarse. El artículo indefinido "un" no marca ausencia de determinación ni particularidad o individualidad; es un encuentro de cualquier "un" con cualquier otro "un", encuentro singular ni particular ni universal (Kohan, 2009). Devenir-niño es encontrarse y situarse intensivamente en el mundo; un salir siempre de "su" lugar y situarse en otros, desconocidos, inusitados, inesperados; es algo sin pasado, presente o futuro; algo sin temporalidad cronológica, más con geografía, intensidad y dirección propias (Deleuze \& Guattari, 1997).

La actitud investigativa como condición crítico- reflexiva. En este tipo de actitud investigativa, prescinden dos fenómenos sustanciales e indisolubles como los son la reflexión y la crítica: la reflexión aparece

2 Aión designa la intensidad del tiempo de la vida humana, un destino, una duración, una temporalidad no numerable ni sucesiva, sino intensiva. Si chrónos es límite, aión es duración. 
como un elemento que se utiliza para designar el modo como la persona humana tiene un cierto conocimiento de sí misma, ese autoconocimiento aparece como posibilitado por algo análogo al proceso en el que la luz física es arrojada hacia atrás por una superficie reflectante (Larrosa, 1995). La reflexión es una mirada constante sobre los actos, los pensamientos, las formas de estar en y con el mundo, de modo tal, que como una actitud en la investigación en la formación escolar, es una constante atención a los fenómenos circundantes en el proceso investigativo, es el despertar de los sentidos sobre lo que se hace en la indagación. Al dirigir sobre sí mismo una mirada irónica que desvela, desenmascara e ilumina lo oculto, antes de poseer la intención de destruirse, es por el contrario, de servirse y de utilizar las herramientas sociales del sujeto para convertirlo en un sujeto mejor (Bourdieu, 2001).

Este autoexamen es una condición de "autovigilancia" particularmente profunda sobre los aspectos en que debe moverse el investigador escolar, donde se deben considerar y monitorear las distintas cartografías que se diseñan durante la indagación. La reflexión depende del pliegue que se hace hacia uno mismo y de los dispositivos generales de la visibilidad. En segundo lugar, obedece a la puesta en obra de dispositivos específicos para la autoobservación. La persona investigadora que reflexiona, que se analiza a sí misma durante el proceso de investigación, se constituye como un conjunto de mecanismos en los que se observa, se forma en el sujeto de la autoobservación, y se consolida a sí mismo desde su propia visión (Larrosa, 1995).

La posición crítica en la actitud investigativa en escolares, como producto de la reflexión, se caracteriza por buscar el cuestionamiento y transformación permanente de la organización de las formas de hacer investigación en la escuela. Este escenario busca hacer de la mente del escolar una entidad más precisa para la comprensión de argumentos en la praxis investigadora. La posición crítica en la actitud investigativa, está mediada en la formulación de preguntas significativas y relevantes que empiecen a construir criterios para sus juicios al tiempo que se esfuerza por razonar con coherencia, será, sin duda, alguien que habrá de plantear nuevos retos a sus maestros y al sistema educativo (Pineda, 2004).

Las posiciones críticas del escolar en la actitud investigativa son principalmente la posibilidad de razonar, examinar, autocorregirse y ser sensible al contexto; es una condición indisoluble entre el razonamiento 
y la construcción de juicios, analogía que se construye en las distintas circunstancias que se le pueden presentar en su trayecto investigativo, donde las distintas herramientas de juicio y discernimiento, permiten darle mayor número de argumentos a los educandos, en términos de lo que desean hacer explícito a través de los juicios y acciones consigo mismo y con los otros (Lipman ,1998).

El espíritu crítico es portador de un sentido de insatisfacción permanente ante toda respuesta que invita a examinar tanto los procesos como los resultados (Pineda, 2008). Esta experiencia investigativa basada en la crítica es, como dice Kant, una condición orientada por la libertad de pensar que lleva al desarrollo del ingenio, donde las críticas al ser comunicadas e intercambiadas con otros, forman la condición para que sean asumidas por la sociedad y le permitan al sujeto la comprensión de las contradicciones del mundo (Henao, 2001).

La reflexión y la crítica como realización de los principios que orientan la actitud investigativa, son la forma en que el joven hace realidad su modo de pensar y transformar el mundo, son procesos de comparación de la consciencia y el proceso de formación del conocimiento, como un hábito consciente de la correlación entre el sujeto y el entorno (Cubillos \& cols., 2001).

La actitud investigativa como conocimiento de sí mismo. El acto de conocerse a sí mismo, es el inicio de la experiencia de la vida en relación con la sabiduría, donde la vida se transforma en una obra de arte. El conocerse es la pauta que permite que los sujetos se gobiernen a sí mismos y representen en la praxis sus elecciones y deliberaciones frente al entorno como sujetos libres (Cubillos \& cols., 2001).

El "cuidado o conocimiento de sí" es una práctica reflexiva de la libertad que gira en torno de este imperativo fundamental: "cuídate de ti mismo" (Foucault, 1999). Estudiar la constitución del educando como objeto para sí mismo, es descifrar los procedimientos que se han inducido para la supuesta formación del sujeto investigador, es analizarse, descifrarse, reconocerse dentro del dominio de ser primero investigador de sí mismo. Se trata, en suma, de la historia de la "subjetividad", si se entiende esta palabra como el modo en que el sujeto hace de la experiencia de sí mismo, 
un juego de verdad en el que se está en relación consigo mismo ${ }^{3} \mathrm{El}$ papel básico de la mirada hacia adentro, demuestra el modo como se tiende a comprender la relación del sujeto consigo mismo, como se autoactualiza y renueva sus conocimientos una y otra vez.

El conocimiento de sí mismo en relación con la actitud investigativa, tiene que ver en cómo el individuo que se cuida a sí mismo, termina por gobernarse a sí mismo, y a su vez le da la capacidad de gobernar a los demás. Es decir, hay que ser médico de uno mismo antes de abordar como principio de mediación al otro, es a través del otro que se puede actuar para la constitución de sí como sujeto (Gil, 2001). La actitud investigativa en relación con el conocimiento de sí mismo, se constituye en tres formas de representación, la actitud general, como una relación con uno mismo, con nosotros y con el mundo; la actitud como una mirada, una vigilancia sobre lo que uno piensa y lo que acontece en el pensamiento, y la actitud como una forma de transformarse como práctica y ejercicio de todo individuo.

El conocimiento de sí mismo consiste en la tarea de realizar de la vida una permanente crítica del ser histórico, es una actitud frente a sí mismos y aquello que se piensa, se siente y se hace. Por tanto, esta consideración no consiste en descubrir lo que se es, sino en rechazar el tipo de individualidad que se ha impuesto durante siglos, por medio de los distintos dispositivos, entre éstos el pedagógico (Giraldo, 2011). Para decirlo más claramente, el conocimiento de sí, implica resistir a los poderes que intentan controlar, clasificar y normalizar la vida (Schmid, 2002).

Las "prácticas de sî" o el "ejercicio de sî", a través de la actitud investigativa es una forma bajo la cual desde el dispositivo pedagógico, el individuo escolar se transforma y accede a un cierto modo de ser, a un "gobierno de sí". Es aquí donde la estética de las formas investigativas en la escuela, se vinculan con una manera de resistencia a los mecanismos de sujeción impuestos durante muchos años por los paradigmas de la ciencia, especialmente en la relación de lo que se quiere ser y lo que otros quieren que uno sea. Mediante el conocimiento de sí, el educando inspecciona sus propios obstáculos, este reconocimiento es una noción fundamentalmente política, que tiene que ver con el principio de la existencia en toda su perfección posible, es jugarse la existencia en la verdad de lo que uno dice y piensa (Giraldo, 2011).

3 La cita es de un texto que Michel Foucault escribió bajo el seudónimo de Maurice Florence. 


\section{Propiedades pedagógicas de las actitudes investigativas}

Las actitudes investigativas se definen como una diversidad de cuestiones y movimientos pivotantes, que por medio de la interacción constante de sí mismo, la relación con la experiencia crítico-reflexiva y el devenir con la infancia, tienen como sentido promover otra conceptualización de lo que se denomina hoy como ciencia y formación investigativa. Estas actitudes son de por sí una condición de multiplicidades, estados que llevan a la superación de las dicotomías y jerarquías del pensamiento y la enseñanza arborescente ${ }^{4}$ establecidas por la ciencia, y reproducidas por los educandos y los maestros. Las actitudes investigativas, son una posibilidad de direcciones cambiantes que no tiene ni principio ni fin, siempre tiene un medio por el que crecen y se desbordan como líneas de fuga que se tocan, se cruzan, se enredan, se confunden, que mutan al cambiar de naturaleza o de contexto.

El dinamismo de las actitudes investigativas en la formación escolar es un nomadismo ${ }^{5}$ que libera a los educandos de la pretensión unificadora, totalizadora; es una fuerza de resistencia, un "exorcizar la vergüenza" (Kohan, 2009). El sentido pedagógico nómada lleva a pensar cómo los elementos sistemáticos en la formación investigativa, pueden desinstalarse de todos los preceptos que le sostienen, como líneas de afectación entre los fenómenos que convergen con los individuos, movimientos que reconstruyen y deconstruyen la participación de los sujetos, como organismos activos en la construcción y agenciamiento ${ }^{6}$

4 El pensamiento arborescente es aquel que opera por la jerarquización, el orden, el poder y la centralidad, "son estructuras y relaciones binarias y biunívocas o sea, que el árbol siempre tiene algo de genealógico, como un estatuto del estado de las cosas" (Deleuze \& Guattari, 1997). El árbol o la raíz inspiran una imagen del pensamiento que no cesa de imitar a partir de una unidad superior, de centro o de segmento.

5 Ser nómada es alcanzar una velocidad, un movimiento absoluto, se puede ser nómada sin salir del lugar.

6 Por agenciamiento, Guattari y Rolnik se refieren a una "noción más amplia que la de estructura, sistema, forma, etc. Un agenciamiento incluye componentes heterogéneos, tanto de orden biológico como social, maquínico, gnoseológico, imaginario" (2005). $\mathrm{Al}$ contrario de las estructuras, que "están siempre ligadas a condiciones de homogeneidades", los agenciamientos son co-funcionales, son una simbiosis (Deleuze \& Parnet, 1987: 52). El agenciamiento es una multiplicidad que incluye tanto líneas molares como moleculares; esta es "la verdadera unidad minima" que ellos proponen en lugar de la palabra, del concepto o del significante (Deleuze \& Parnet, 1987: 51). Deleuze planteará en sus escritos que "la unidad real mínima no es la palabra, ni la idea o el concepto, ni tampoco el significante. La unidad real mínima es el agenciamiento". El profesor Humberto Sabatini (2001) planteará en sus clases: "Todo agenciamiento es colectivo y pone en juego poblaciones, multiplicidades, afectos, intensidades, territorios. Siempre hablamos, accionamos y pensamos desde un agenciamiento, es la línea imperceptible que atraviesa las ideas, los cuerpos, los elementos en juego, es el entremedio, que sostiene todas las relaciones". 
con y en los otros. Por eso, ser nómada como parte de dispositivo pedagógico de las actitudes investigativas, es el acontecimiento (acontecer) y la experiencia (experienciar), como verbos en infinitivo y no conjugados o sustantivos (Kohan, 2009) que están directamente relacionados con las actitudes investigativas como forma de vida.

Las actitudes investigativas pueden considerarse como elementos de mutantes intensidades, al plano de lograr una relación íntima y solidaria con lo que se está investigando, con los otros como iguales, como sujetos educandos inacabados que quieren aprender, tomar riesgos, transformarse $\mathrm{y}$ transformar el mundo. El que investiga necesita estar abierto al otro, a lo otro, a sí mismo y a la transformación permanente. Igualmente, es "estar ahí", en el lugar del otro y lo otro, sabiendo que aunque es imposible lograr tal condición, por lo menos sabe que se intenta (Messina, 2011).

Las actitudes investigativas dentro de la formación investigativa, están situadas en la urdimbre de elementos que interactúan entre sí, haciendo que los educandos tengan formas variadas de indagar y transformar la realidad particular en la cual están inmersos. La ontología compleja de las actitudes investigativas dentro del aula, no es fácilmente perceptible a la vista de la pedagogía tradicional, este terreno de la subjetividad está asociado a la interpretación del mundo, es donde el joven investigador coloca un punto de vista más abierto frente a la investigación y a sí mismo, desde el reconocimiento de los contextos y procesos cultural e históricamente constituidos. Las actitudes investigativas del educando parten de interpretar el significado del mundo, y cómo sus valoraciones transforman el entorno cultural, social, investigativo y educativo.

Las propiedades pedagógicas de las actitudes investigativas parten de fenómenos que se interconectan independiente de su genealogía; es en la práctica donde encuentran su convergencia, pero a la luz de la investigación no presentan ningún orden jerárquico, ninguna actitud antecede ni precede a la otra, simplemente habitan en cada sujeto juvenil y se presentan de acuerdo a la necesidad o intención del educando en su proceso indagador. Las actitudes investigativas son puntos que se encuentran, que no se anulan, se diferencian, pero en su conexión, hacen de estas actitudes formas cada vez más distintas.

Igualmente, las actitudes investigativas dentro de los elementos pedagógicos no tienen tiempo ni espacio, y menos aún, una institución que las moldee; 
las actitudes son cambiantes y se transforman en la interacción mediada con el entorno y los otros, estas condiciones y su representación son únicas, haciendo de la realidad educativa de los educandos un elemento complejizado. Este tipo de actitudes y sus conexiones no pueden ser catalogadas como únicas o verdades absolutas, se movilizan por un espacio abierto, un espacio nómada que está marcado por trazos que se borran, y pueden ser irregulares en el contexto educativo.

Las actitudes investigativas no se encausan a un fenómeno único del aula, o se limitan a un espacio de cuatro paredes, son un fenómeno extramural, que lleva a que los estudiantes estén en permanente cuestionamiento del entorno, de las cosas que suceden y de sí mismos. Igualmente estas actitudes no semejan, ni reproducen los modelos investigativos tradicionales, contrariamente, los transforman en cada encuentro del educando investigador con su realidad; a la par, no desconocen estas estructuras culturales del saber, las distinguen, se insertan en ellas vivenciándolas, y las modifican. Condiciones como el "emocionar y experienciar" cada fenómeno, hacen que este sitio de la reproducción, sea cambiado por la creación, la innovación en el contexto de la sensibilidad.

\section{A modo de conclusión}

En el afán constante de protestas frente a los sistemas que se imponen en el campo pedagógico-educativo, pueden existir formas de cambiar la realidad. Algunos, como los paradigmas tradicionales y positivistas en la formación educativa, últimamente han querido cambiar el contexto con un estilo de fuerza basada en la imposición y homogeneización de los conocimientos a través de los distintos dispositivos, entre ellos la escuela.

Los sujetos escolares, dentro del medio pedagógico e investigativo que se les inculque, a través de las distintas relaciones con el entorno, cambian la realidad con la creación y la indagación. Se cambia la realidad inventando otra realidad, se cambia, transformando las reglas del juego. Las actitudes investigativas de los educandos, son un modo de enfrentar con más oportunidad las contradicciones que hacen parte de la lógica de lo humano y lo adulto. La clave está en desdibujar los roles habituales, la horizontalidad propia de los adultos, desde las particulares percepciones de los jóvenes y sus modos de pensar, de reflexionar, desde sus imaginarios y su sensibilidad. 
Por medio de esta reflexión teórica se puede analizar que el tipo de actitud investigativa constituida en el centro de la formación positivista tradicional se caracteriza y se representa principalmente en la búsqueda de razones asociadas a cuestionamientos de causa y efecto, la comprobación y refutación de hechos, y el desarrollo de la investigación por medio de normas y reglas "virtuosas", que aprueban un orden sistemático de la investigación. Igualmente, el análisis de este tipo de actitud devela que los estudiantes desde esta posición investigativa, mediante la réplica de los paradigmas para investigar, perpetúan, "cuidan y mantienen" el statu quo de la investigación positivista tradicional. Los educandos por medio de este tipo de actitud investigativa pueden generalizar y explicar de forma causal la formación del conocimiento, haciendo a su vez un trabajo inhibitorio sobre su capacidad creativa.

Este tipo de actitud basada en la ciencia explicativa, está sujeta a imposibilitar pensar a los sujetos, y solo reafirma la incapacidad de los educandos de no comprender por sí mismos la realidad, sujetando a los alumnos a utilizar medios y métodos organizados de indagación de la verdad. Igualmente, lleva a que los estudiantes en su quehacer investigativo, estén en la constante lucha de aprobar y certificar los conocimientos constituidos en el proceso de investigación. Este tipo de actitud positivista tradicional, es una condición culturalmente constituida, se ha mantenido en la jerarquía del saber como un elemento hegemónico, jerárquico, organizado e impositivo, independiente del entorno o del campo disciplinar en que estén inmersos los estudiantes.

La actitud investigativa como experiencia de la infancia es una forma de vivenciar muchos de los fenómenos y estados experimentados en esta condición de la vida, resignificando la infancia y la experiencia infantil mediante el devenir-joven. Esta experiencia se representa a través de la imaginación, la curiosidad, la creatividad y la incertidumbre que pueden expresar los estudiantes cuando están investigando. Este tipo de actitud investigativa posee una relación con la forma política de los sujetos dentro del aula, se vuelve un medio de cuestionamiento del orden por medio de la resistencia, e implica principalmente la invitación a no replicar las metodologías cultural e institucionalmente constituidas.

La actitud investigativa como condición crítico-reflexiva es la opción de los educandos de formular cuestionamientos y preguntas para reinterpretar y apreciar el entorno de un modo distinto; además este 
constante cuestionamiento les permite a los educandos despertar el sentido de la reflexión, como primera forma de transformar la realidad. Del mismo modo, este tipo de actitud es una forma de examinación e inspección sobre la forma como se está construyendo la propia formación investigativa. Esta actitud es un medio para la transformación personal y social, vinculada al encuentro de los límites entre los distintos paradigmas, especialmente en relación al posicionamiento positivista tradicional.

Frente a la actitud investigativa como conocimiento de sí, se puede analizar que este tipo de actitud permite vincular la vida del educando al conocimiento, especialmente en la conciencia que empieza a tener el estudiante sobre lo que hace. Igualmente la actitud investigativa como conocimiento de sí mismo, es una forma por la cual el estudiante investigador se inventa, se elabora a sí mismo. Este tipo de actitudes investigativas, cuando son reconocidas en los estudiantes, son absolutamente elementos transformadores del aula y de los paradigmas impuestos en la formación investigativa, a través de la visibilidad de las nuevas conexiones que hacen los educandos sobre el conocimiento, la experiencia, la formación investigativa y la propia vida.

En síntesis, se puede analizar que las actitudes investigativas no parecen tener orden o jerarquía en los educandos, son movedizas entre sí; estas uniones son un eje particular y subversivo de modificar las condiciones pedagógicas del aula frente a la formación en investigación y el sentir investigativo de los jóvenes. Estas actitudes simplemente habitan y se ligan durante la experiencia investigativa, igualmente no son temporales, moran en los estudiantes creando nuevas y variadas formas de ver el mundo, además que éstas no solo se aplican en el contexto educativo, las actitudes investigativas están presentes en todo momento, y lo más importante: las actitudes investigativas en los estudiantes, son variadas y son contrarias a reproducir las formas impuestas y anquilosadas de investigación.

\section{Referencias}

Besley, T. \& Marshall, J. (2005). 'Foucault and Disciplining the Self'. Educational Philosophy and Theory [37, 3, 309-315].

Bourdieu, P. (2001). El oficio del Científico: Ciencia de la ciencia y reflexividad. Barcelona: Editorial Anagrama.

Bustamante, G. (1998). ‘¿Se puede formar en la investigación?’ Revista Pedagogía y Saberes [11, 17-23]. Bogotá: Universidad Pedagógica Nacional. 
Cubillos, S. \& cols. (2001). Educar para pensar, La actitud filosófica: un concepto de formación. Cali: Univalle.

Deleuze, G. \& Guattari, F. (1997). Mil Mesetas. Capitalismo y esquizofrenia. Valencia: Pre-Textos.

Deleuze, G. \& Parnet, C. (1987). Diálogos. Valencia: Pre-Textos.

Díaz, B. F. \& Rigo, M. A. (2000). 'Formación docente y educación basada en competencias'. Valle, M. A. [coord.]. Formación en competencias y certificación profesional. Pensamiento Universitario. Tercera época. México: CESU/UNAM.

Flórez, R. (1998). Hacia una pedagogía del conocimiento. Bogotá: McGraw Hill.

Florence, M. “Foucault, M.” (1984). Dictionaire des Philosophes [297-298]. París.

Foucault, M. (1981). Tecnologías del yo y otros textos afines. Barcelona: Editorial Paidós.

. (1999). Estética, Ética y Hermenéutica. Obras esenciales. Vol. III. Barcelona: Paidós Ibérica. . (2005). Vigilar y Castigar. España: Siglo XXI Editores.

Freire, P. (1992). Pedagogía del Oprimido. México: Siglo XXI Editores.

GIL, M. (2001). 'Filosofía y Actitud Pedagógica'. Cubillos, S. Educar para pensar, La actitud filosófica: un concepto de formación [87-97]. Cali: Univalle.

Gimeno, J. \& Pérez, A. (1992). Enseñanza para la comprensión. Comprender y transformar la enseñanza. Madrid: Morata.

Giraldo, R. (2011). 'Modernidad y parrhesía. Michel Foucault y la cuestión de la resistencia como éthos'. Revista Estudios de Filosofía [137-147]. Antioquia: Universidad de Antioquia.

Giroux, H. (2004). Teoría y resistencia en educación: Una pedagogía para la oposición. México: Siglo XXI Editores.

GubA, E. \& Lincoln I. (2000). 'Paradigmas en competencia en la investigación cualitativa'. Denman, C. A \& Haro, J. A. [ed.]. Por los rincones. Antología de métodos cualitativos en investigación social [113-145]. Sonora: El Colegio de Sonora.

Henao, L. (2001). 'Una nueva actitud para la educación del tercer milenio'. Cubillos, S. Educar para pensar, La actitud filosófica: un concepto de formación [39-65]. Cali: Univalle.

Kant, E. (1980). Réflexions sur l'education. París: Vrin.

Kincheloe,L. (2005). 'On to the Next Level: Continuing the Conceptualization of the Bricolage'. Magazine Qualitative Inquiry [11, 3, 323-350]. Sage Publications.

Kohan, W. (2009). Filosofía e Infancia. México D.F.: Progreso. 
Larrosa, J. (1995). 'Tecnologías del yo y educación. Notas sobre la construcción y la mediación de la experiencia de sí.. Larrosa, J. [Ed.]. Escuela, poder y subjetivación [259-232]. Madrid: La Piqueta. . (2003). La experiencia y sus lenguajes. [Conferencia]. Recuperado en septiembre de 2011 de www.me.gov.co.ar/curriform/publica/ oei_20031128/ponencia_larrosa.pdf

Liebel, M. (2007). 'Jóvenes investigadores'. Revista Encuentro [78, 6-18]. Argentina: Pontificia Universidad Católica de Argentina.

Lipman, M. (1998). Pensamiento Complejo y Educación. Madrid: Ediciones de la Torre.

Meirieu, P. (1998). Frankenstein Educador. Barcelona: Editorial Laertes S.A. Melich, C. (2002). Filosofía de la Finitud. Barcelona: Editorial Herder.

Messina, G. (2011). 'Investigación y Experiencia'. Revista Praxis E Saber [4, 61-75]. Tunja: Universidad Pedagógica y Tecnológica de Colombia, Maestría en Educación.

Moreno, M. (2005). 'Potenciar la educación. Un currículum transversal de formación para la investigación'. Revista Electrónica Iberoamericana sobre Calidad, Eficacia y Cambio en Educación (REICE) [3, 1]. Recuperado de http://redalyc.uaemex.mx/pdf/551/55130152.pdf

PINEDA, D. (2004). Filosofía para jóvenes, el ABC. Bogotá. Editora Beta. - (2008). La construcción del oficio investigador: Una perspectiva Sherlokiana. Bogotá: Meditaciones Sherlokianas.

Quiceno, C. (1988). Corrientes pedagógicas en el siglo XX en Colombia. Bogotá: Universidad del Valle.

RANCIĖRE, J. (2007). El maestro ignorante. Cinco lecciones para la emancipación intelectual. Buenos Aires: Libros del Zorzal.

Rodríguez, A. (1976). Psicología social. México: Trillas.

Schmid, W. (2002). En busca de un nuevo arte de vivir. La pregunta por el fundamento y la nueva fundamentación de la ética en Foucault. Cano, G. [Trad.]. Barcelona: Pre-Textos.

Secord, P. \& Backman, C. (1964). Social Psychology. New York: McGrawHill.

Valera, G. (2001). 'Escuela, alteridad y experiencia de sí: La producción pedagógica del sujeto'. Revista Educere [5, 13, 25-29]. Mérida: Universidad de Los Andes. 